\title{
Caries incidence in restorations of shortened lower dental arches - RBBs versus RPDs
}

\author{
Caries incidence following restoration of shortened lower dental arches in a randomized controlled trial \\ N. J. A. Jepson, P. J. Moynihan, P. J. Kelly, G. W. Watson and J. M. Thomason Br Dent J 2001; 191: 140-144
}

\section{Context}

Removable partial dentures used to restore the shortened lower dental arch may adversely affect the remaining natural teeth and are associated with a low prevalence of use.

\section{Objective}

To report the findings for caries incidence 2 years after restoration of lower shortened arches with bilateral cantilever resin-bonded bridges (RBBs) and conventional partial dentures (RPDs).

\section{Design}

Randomised controlled trial.

\section{Setting}

Secondary care

\section{Patients}

25 male and 35 female subjects of median age 67 years were randomly allocated to 'bridge' and 'denture' treatment groups of 30 patients each matched for age and sex. Caries incidence was recorded during dental examinations 3 months, 1 and 2 years after insertion of new lower prostheses.

\section{Interventions}

Cantilever RBBs and conventional RPDs with cast metal frameworks.

\section{Results}

There was a highly significant difference in the frequency of new caries lesions, 11 and 51 in the bridge and denture groups respectively $(P<0.01) .20$ out of 27 bridge patients and 9 of 23 denture patients had no caries experience. Multivariate modeling identified treatment group as the only significant predictor of caries occurrence.

\section{Conclusions}

Two years after restoration of lower shortened arches for an elderly sample of patients, there was a significantly greater incidence of new and recurrent caries lesions in subjects restored with RPDs compared with cantilever RBBs.

\section{Comment}

7 his paper reports on the results of a care-

fully controlled study of the effects of two treatments on a population of median age of 67 years.

The importance of attention being paid to patients in this age group has been emphasised recently in a group of papers describing some of the findings from the 1998 Adult Dental Health Survey and published in the British Dental Journal. ${ }^{1,2}$ Results from the Survey highlighted the increased retention of natural teeth into old age, the exposure of vulnerable root surfaces and the still widespread problem of relatively poor oral hygiene. Thus, the provision of a prosthesis, whether fixed or removable, would seem to carry a degree of risk. The authors set out to quantify one aspect of that risk, namely caries. The first decision regarding the treatment of a depleted lower dentition must surely be 'to treat or not to treat'. The benefits of the shortened dental arch have been reported widely. If such an arch can be established without having to provide a prosthesis, so much the better. The results reported in this paper would seem to support the concept that, if more function is required, there would appear to be a benefit, with respect to caries prevention, in providing simple cantilever resin-bonded bridges rather than more extensive RPDs. It has to be said, though, that the authors have come to this conclusion by comparing the fixed restorations with RPDs, which mostly include a connector that covers the vulnerable gingival margins and therefore increase the risk of damage to the oral tissues, especially if the plaque control of the denture is substandard.

If, for good reasons, we have to provide a lower bilateral free end saddle RPD it is comforting to recall the work of Bergman et al. ${ }^{3,4}$ which showed that favourable outcomes could be achieved over long periods of time if the patients maintained good plaque control of mouth and dentures and if the dentures were designed according to particular criteria which included noncoverage of gingival margins.

The authors are apparently also looking at the effect of the two types of prosthesis on diet, patient satisfaction and periodontal status as well as investigating survival time. I, for one, look forward with interest to future reports.

\section{R. M. Basker}

Emeritus Professor, University of Leeds

1 Nunn J et al. The condition of teeth in the UK in 1998 and implications for the future. Br Dent J2000; 189: 639-644.

2 Pine C M et al. Dental Restorations in adults in the UK in 1998 and implications for the future. Br Dent J2001; 190: 4-8.

3 Bergman B, Hugoson A, Olsson C-O. Caries, periodontal and prosthetic findings in patients with removable partial dentures: A ten-year longitudinal study. J Pros Dent 1982; 48: 506514.

4 Bergman B, Hugoson A, Olsson C-O. A 25 year longitudinal study of patients treated with removable partial dentures. J Oral Rehabil 1995; 22: 595-599. 